\title{
Composition of pathogenic bacteria of chronic obstructive pulmonary disease patients and drug resistance of gram-negative bacilli for various antibiotics.
}

\author{
Lin Chen*, Zheng-Liang Xie, Yang Yang \\ Department of Respiratory Medicine, Sichuan Provincial People's Hospital, PR China
}

\begin{abstract}
Objective: In order to analyze the composition and characteristics of pathogens resistance, provide reference for rational use of antibiotics, clinical bacteria isolated from COPD patients in our hospital was investigated.

Methods: The 710 patients with chronic obstructive pulmonary disease were collected from August 2010 to February 2014. All patients required to multiple gargle with water or hydrogen peroxide before collecting specimens, ensuring a sterile culture spit sputum specimen box. Susceptibility was determined by the United States Committee for Clinical Laboratory Standards (NCCLS) developed by KB.

Results: Gram-negative bacteria as the main species which 813 species were isolated from 710 cases of patients with chronic obstructive pulmonary disease. Pseudomonas aeruginosa based. Gram-positive bacilli was in second place, Staphylococcus aureus, fungi ranks No. 3, Gram-negative bacilli species number and composition were significantly higher than that of Gram-positive bacteria and fungi, and the difference was statistically significant $(\mathbf{p}<0.05)$. Pseudomonas aeruginosa and Acinetobacter baumannii resistant to cefazolin higher rate, the difference was statistically significant $(p<0.05)$.

Conclusion: Gram-negative bacteria are the main pathogens, and resistance was so strong. So we need to constantly monitor the bacteria resistance, and to guide rationally clinical medicine.
\end{abstract}

Keywords: COPD, Bacteria, Drug resistance, Susceptibility testing.

Accepted on December 01, 2017

\section{Introduction}

In recent years, because of abuse of antibiotic drugs, the category, distribution and drug resistance of pathogenic bacteria causing lower respiratory infection have changed greatly [1] and great difficulties have been caused for clinical administration and treatment guidance. As a major common disease for lower respiratory infection, the chronic obstructive pulmonary disease (COPD) includes chronic bronchitis and pulmonary edema. In order to solve composing complexity, non-uniform distribution and rising drug resistance of pathogenic bacteria and low immunity of patients, our hospital selected 871 COPD patients, who took cures in our hospital from August 2012 to December 2016, sampled and cultivated sterile sputum through reasonable measures and analyzed the composition and drug resistance of pathogenic bacteria in patients' sputum to give scientific and reasonable guidance for clinical administration.

\section{Data and Method}

\section{General materials}

Our hospital selected 871 COPD patients, who took cures in our hospital from August 2012 to December 2016. All patients complied with COPD diagnosis standard of the national regulation, including 482 males and 228 females with the age of $45 \sim 80$ and the mean age of $(53.8 \pm 8.1)$. Their clinical conditions were various degrees of cough, fever, expectoration and even gasp and respiratory difficulty and some patients had concomitant edema of lower extremity, varicosity, cyanosis, etc. Among them, there were 395 patients with diabetes, hypertension, renal insufficiency, coronary heart disease, cerebral haemorrhage or other diseases, 279 patients with chronic bronchitis, 19 patients with bronchiectasis and 17 patients with bronchial asthma.

\section{Collection of specimens}

The patients were selected according to the Hospital Infection Diagnosis Standard issued by the Ministry of Health. When brushing teeth in the morning, the patients complying with the selection standard spat up the first mouthful of phlegm, put the second mouthful of phlegm into the sterile pot and immediately sent it for testing. Strain was separated and identified according to the national operating procedures of clinical examination. Within $1 \mathrm{~h}$, the collected specimens were inoculated on chocolate agar plate, sheep blood agar plate and MacConkey agar plate. The specimens could also be collected by cultivation with a bronchoscope and then incubated in $\mathrm{CO}_{2}$ incubator under $35^{\circ} \mathrm{C}$. After cultivation, identification and smearing were carried on as per relevant conditions specified. 
It was observed under a microscopy: number of squamous cell $<10$, number of leucocyte $>25$, or squamous cell: leucocyte $<1: 2.5$, in the low power field, and such relevant specimens were considered as eligible ones with less pollution and could be incubated and cultivated. The cultivated and separated phlegm pathogens with consistency $\geq 10^{7} \mathrm{cfu} / \mathrm{ml}$ were recognized as the malignant bacteria; those with the consistency $\leq 10^{4} \mathrm{cfu} / \mathrm{ml}$ were regarded as pollutant bacteria and should be discarded. If the consistency of phlegm bacteria was $10^{4}-10^{7} \mathrm{cfu} / \mathrm{ml}$, the specimens should be cultivated again, until the same pathogens with the consistencies of $10^{5}-10^{6}$ $\mathrm{cfu} / \mathrm{ml}$ were obtained for successively 3 times and then regarded as the malignant bacteria. Vitek32 bacteria identifier produced by BIOMERIEUX was used for identifying bacteria species.

\section{Susceptibility test}

The susceptibility test was carried out for all bacteria obtained from separation by the K-B paper strip method. The culture medium of the susceptibility test was composed of resin and defibrated sheep blood provided by BIOMERIEUX and the bacteria included Pseudomonas aeruginosa, Acinetobacter baumannii, Escherichia coli, Staphylococcus aureus, Candida albicans, etc. The paper strip contained cefazolin, ampicillin, gentamycin and so on. The results of susceptibility test were judged as per the standards of the National Committee for Clinical Laboratory Standards (CLSI/NCCLS).

\section{Observation indices}

The separated 813 bacteria were recorded and could be mainly divided into three categories of gram-negative bacilli, grampositive bacilli and fungi, each of which the main categories were listed out, respectively. Then, the drugs, which could be resisted by gram-negative bacilli and gram-positive bacilli were, were explored and recorded and the resistance rates were analyzed and recorded.

\section{Statistical treatment}

SPSS 17.0 statistics software was used in analysis and the measurement data were expressed in mean value \pm standard deviation. After homogeneity test for variance and normal distribution test, t-test was carried out for two independent samples in the sample group and the chi-square test was adopted to compare the enumeration data.

\section{Results}

\section{Composition and distribution of pathogenic bacteria}

Totally, 813 species were separated from 710 patients with chronic obstructive pulmonary diseases and divided into three categories of gram-negative bacilli, gram-positive bacilli and fungi. The analysis results of different species were shown in Table 1. The species of gram-negative bacilli were obviously higher than those of gram-positive bacilli and fungi, of which the difference was statistically significant $(p<0.05)$. The constituent ratio of gram-negative bacilli species was obviously higher than those of gram-positive bacilli and fungi, of which the difference was statistically significant $(p<0.05)$.

Table 1. Composition of Pathogenic Bacteria Infected by 170 COPD Patients.

\begin{tabular}{|c|c|c|}
\hline Type & $\mathrm{n}$ & Constituent ratio $/ \%$ \\
\hline Gram-negative bacilli & 554 & 68.1 \\
\hline Pseudomonas aeruginosa & 125 & 15.3 \\
\hline Escherichia coli & 118 & 14.5 \\
\hline Acinetobacter baumannii & 82 & 10.1 \\
\hline Klebsiella pneumoniae & 72 & 8.8 \\
\hline Serratia marcescens & 65 & 8.0 \\
\hline E. cloacea & 45 & 5.6 \\
\hline Other bacilli & 47 & 5.8 \\
\hline Gram-positive bacilli & 179 & 22.1 \\
\hline Staphylococcus aureus & 59 & 7.2 \\
\hline Streptococcus pneumoniae & 48 & 5.9 \\
\hline Staphylococcus epidermidis & 38 & 4.7 \\
\hline Enterococcus & 34 & 4.3 \\
\hline Fungi & 80 & 9.8 \\
\hline Candida albicans & 49 & 6.1 \\
\hline Other bacteria & 31 & 3.7 \\
\hline
\end{tabular}

Note: compared with gram-positive bacilli and fungi, the species and constituent ratio of gram-negative bacilli are $p<0.05$.

\section{Analytic results of pathogenic bacteria's drug resistance}

A total of 5 common antibacterial drugs were used to analyze gram-negative bacilli of main malignant bacteria, of which the drug resistance of 3 gram-negative bacilli (Pseudomonas aeruginosa, Escherichia coli and Acinetobacter baumannii) was mainly researched, with the results shown in Table 2. It could be seen that the resistance of Escherichia coli to piperacillin was obviously higher than those of Pseudomonas aeruginosa and Acinetobacter baumannii, of which the difference was statistically significant $(\mathrm{p}<0.05)$; the resistance of Acinetobacter baumannii to Augmentin was obviously higher than those of Pseudomonas aeruginosa and Escherichia coli, of which the difference was statistically significant $(\mathrm{p}<0.05)$; the resistance of Escherichia coli to cephazoline was obviously lower than those of Acinetobacter baumannii and Pseudomonas aeruginosa, of which the difference was statistically significant $(\mathrm{p}<0.05)$; the resistance of Pseudomonas aeruginosa and Escherichia coli to cefotaxime was obviously higher than that of Acinetobacter baumannii, of which the difference was statistically significant $(\mathrm{p}<0.05)$; the resistance of Acinetobacter baumannii to Ciprofloxacin was obviously lower than those of Pseudomonas aeruginosa and 


\section{negative bacilli for various antibiotics}

Escherichia coli, of which the difference was statistically significant $(\mathrm{p}<0.05)$; the resistance of Escherichia coli to Ciprofloxacin was obviously higher than that of Pseudomonas aeruginosa, of which the difference was statistically significant $(\mathrm{p}<0.05)$.

Table 2. Drug Resistance of Main Gramnegativebacilli.

\begin{tabular}{llll}
\hline Antibiotics & $\begin{array}{l}\text { Pseudomonas } \\
\text { aeruginosa } \\
\text { Strain Resistance }\end{array}$ & $\begin{array}{l}\text { Acinetobacter } \\
\text { baumannii } \\
\text { Strain Resistance }\end{array}$ & $\begin{array}{l}\text { Escherichia coli } \\
\text { Strain Resistance }\end{array}$ \\
\hline Piperacillin & 3830.6 & 1315.9 & 9580.3 \\
\hline Augmentin & 3930.7 & 5373.7 & 2117.9 \\
\hline Cephazoline & 11390.6 & 82100 & 7261.3 \\
\hline Cefotaxime & 7963.5 & 2226.8 & 6758.6 \\
\hline Ciprofloxacin & 6249.7 & 89.8 & 10690.3 \\
\hline
\end{tabular}

\section{Discussion}

The morbidity rate of COPD was increasing with years and the treatment should mainly focus on decrease of lung functions, promotion of patients' immunity and enhancement of lung functions. Successive mutation of pathogenic bacteria has resulted in more complex species and higher drug resistance, finally making clinical treatment difficult. Therefore, in the process of treatment, the important section, which cannot be ignored at least, should be infection of pathogenic bacteria [2]. In this study, tens of antibiotics were used to carry out the drug susceptibility test for 813 pathogenic bacteria according to the K-B method specified by the National Committee for Clinical Laboratory Standards (NCCLS). While guaranteeing that the bacteria must be cultivated under a sterile environment, the physician paid attention to sterile operation and improved sterile consciousness during the drug susceptibility test and all testing instruments were sterilized thoroughly. Antibiotics were reasonably selected according to the results of the drug susceptibility test so as to avoid treatment difficulty caused by blind selection [3]. In this study, the drug resistance of 3 gramnegative bacilli (Pseudomonas aeruginosa, Escherichia coli and Acinetobacter baumannii) was researched, from which it was found that the resistances of Pseudomonas aeruginosa and Acinetobacter baumannii to cephazoline were the highest, of which the different was statistically significant $(\mathrm{p}<0.05)$, the Pseudomonas aeruginosa had extreme drug resistance and could not be thoroughly eliminated by the respiratory system, resulting in decrease of patients' lung functions and repeating of illness state [4]. The drug resistance of Escherichia coli to Ciprofloxacin was the highest, of which the different was statistically significant $(\mathrm{p}<0.05)$.

In conclusion, the sick and morbidity rates of COPD, as the most frequent disease for infection of the respiratory, are increasing year by year. Focusing on the key point that pathogenic bacteria is the most important cause for the said disease, this study has discovered composing complexity, strong drug resistance and other features of pathogenic bacteria via bacteria cultivation and drug susceptibility test. If the change in illness state and strain can be predicted from effective clinical data, it will be quite convenient for clinical use of antibacterial drugs [5].

\section{References}

1. Buyck J M, Guénard S, Plésiat P. Role of Mex A B-OprM in intrinsic resistance of Pseudomonas aeruginosa to temocillin and impact on the susceptibility of strains isolated from patients suffering from cystic fibrosis. J Antimicrob Chemother 2012; 67: 771-775.

2. Rothberg M B, Pekow P S, Lahti M. Antibiotic therapy and treatment failure in patients hospitalized for acute exacerbations of chronic obstructive pulmonary disease JAMA 2010; 303: 2035-2042.

3. Joseph NM, Sistla S, Dutta TK. Ventilator - associated pneumonia: A review. Eur J Intern Med 2010; 21: 360-368.

4. Xiao-Shan Zhang, Sha-Ning Yang, Yu-Jing Wang, Xue-xin Yang, Li-Jun Jin and Zhong-Quan Zhou. Therapeutic effect of simvastatin combined with vein infusion of hyperoxic fluid on the cardiac function, respiratory mechanics and airway inflammation factor of patients with chronic obstructive pulmonary disease combined with coronary heart disease. Biomed Res 2017; 22: 10210-10215.

5. Butorac-Petanjek B, Parnham MJ, Popovic-Grle S. Antibiotic therapy for exacerbations of chronic obstructive pulmonary disease (COPD). J Chemother 2010; 22: 291-297.

\section{*Correspondence to}

Lin Chen

Department of Respiratory Medicine

Sichuan Provincial People's Hospital

PR China 